\title{
Enteroparasitoses, anemia e estado nutricional de uma população ribeirinha no estado do Amapá
}

\author{
Enteroparasitosis, anemia and nutritional status of a riverside population in the state of \\ Amapá
}

Enteroparasitosis, anemia e estado nutricional de uma população ribeirinha no estado do Amapá

Risomar Carréra de Menezes Júnior ${ }^{1}$, Carlos Augusto Alves de Lima Junior ${ }^{1}$, Inakê Gomes Marinho', Kelly Huany de Melo Braga ${ }^{1}$, Rosana Oliveira do Nascimento', Tatiana do Socorro dos Santos Calandrini', Débora Prestes da Silva Melo ${ }^{1}$, Benedito Pantoja Sacramento ${ }^{2}$, Rosemary Ferreira de Andrade ${ }^{1}$, Rubens Alex de Oliveira Menezes ${ }^{3 *}$.

\section{RESUMO}

Objetivo: Verificar a prevalência de parasitoses intestinais e sua relação com a anemia e estado nutricional em uma comunidade ribeirinha do Amapá. Métodos: Estudo transversal através de exames coprológicos pelos métodos direto e de Hoffman, questionário estruturado, e coleta de sangue para análise de hemograma e dosagem de ferritina. Os dados foram coletados de 136 residentes da comunidade ribeirinha. Resultados: Demonstraram que 65 indivíduos apresentaram índices hematimétricos sugestivos de anemia, uma prevalência de 47,8\%, destes, 75,4\% (49/65) são crianças e adolescentes e apenas $24,6 \%$ (16/65) maiores de 18 anos. Sobre os valores de índice de massa corpórea (IMC) dos indivíduos anêmicos, observou-se que $55,38 \%$ (36/65) apresentavam-se abaixo do peso, destes, 91,66\% (33/36) eram crianças e adolescentes com idades entre 0 a 15 anos. Os resultados demonstraram uma positividade parasitária de 95,6\% (130/136), tendo o poliparasitismo $59,6 \%$ (81/136) prevalecendo frente ao monoparasitismo $36 \%(49 / 136)$. Para protozoários a prevalência observada foi de $89 \%$ (121/136), e para helmintos de $6,6 \%(09 / 136)$. Conclusão: Com base nos resultados, há uma elevada prevalência de parasitoses intestinais humanas na comunidade estudada, além de alta prevalência de crianças anêmicas e abaixo do peso.

Palavras-chave: Anemia, Déficit nutricional, Parasitoses, Saúde pública, Vulnerabilidade.

\begin{abstract}
Objective: Verify the prevalence of intestinal parasites and their relationship with anemia and nutritional status in a community of Ribeirinha do Amapá. Methods: Cross-sectional study through direct and Hoffman coprological examinations, structured questionnaire, and blood collection for blood count analysis and ferritin dosage. I gave you foram collected from 136 residents of the Ribeirinha community. Results: They showed that 65 individuals had hematimetric indexes suggestive of anemia, a prevalence of $47.8 \%$, of these, $75.4 \%$ $(49 / 65)$ are children and adolescents and only $24.6 \%$ (16/65) over 18 years. years. Regarding the body mass index (BMI) values of anemic individuals, it was observed that $55.38 \%(36 / 65)$ were underweight, of these, $91.66 \%(33 / 36)$ were children and adolescents. aged 0 to 15 years. The results showed a parasitic positivity of $95.6 \%$ (130/136), with polyparasitism $59.6 \%$ (81/136) prevailing over monoparasitism $36 \%(49 / 136)$. For protozoa the prevalence observed was $89 \%$ (121/136), and for helminths $6.6 \%(09 / 136)$. Conclusion: Based on the results, there is a high prevalence of human intestinal parasites in the studied community, as well as a high prevalence of anemic and underweight children.
\end{abstract}

Keyword: Anemia, Nutritional deficit, Parasitosis, Public health, Vulnerability

1 Universidade Federal do Amapá (UNIFAP), Macapá-AP, Brasil.

2 Faculdade Metropolitana da Amazônia (FAMAZ), Belém, Pará, Brasil.

3 Universidade Federal do Amapá (UNIFAP), Macapá-AP. *E-mail: rubens.alex@unifap.br

SUBMETIDO EM: 12/2019

ACEITO EM: 1/2020

PUBLICADO EM: 3/2020 


\section{RESUMEN}

Objetivo: Verifique una prevalência de parasitosis intestinais y su relación con una anemia y un estado nutricional en una comunidad comunidade ribeirinha do Amapá. Métodos: estudio transversal a través de exámenes coprológicos directos y de Hoffman, cuestionario estructurado y recolección de sangre para análisis de recuento sanguíneo y dosificación de ferritina. Se recopilaron datos de 136 residentes de la comunidad ribereña. Resultados: mostraron que 65 individuos tenían índices hematimétricos sugestivos de anemia, una prevalencia del $47.8 \%$, de estos, el $75.4 \%$ (49/65) son niños y adolescentes y solo el $24.6 \%(16 / 65)$ mayores de 18 años. años Con respecto a los valores del índice de masa corporal (IMC) de individuos anémicos, se observó que el 55.38\% (36/65) tenían bajo peso, de estos, el $91.66 \%$ (33/36) eran niños y adolescentes. De 0 a 15 años. Los resultados mostraron una positividad parasitaria del 95.6\% (130/136), con el poliparasitismo $59.6 \%(81 / 136)$ prevaleciendo sobre el monoparasitismo 36\% (49/136). Para los protozoos, la prevalencia observada fue del $89 \%(121 / 136)$ y para los helmintos del 6,6\% (09/136). Conclusión: Según los resultados, existe una alta prevalencia de parásitos intestinales humanos en la comunidad estudiada, así como una alta prevalencia de niños anémicos y con bajo peso.

Palabra clave: Anemia, Déficit nutricional, Parasitosis, Salud pública, Vulnerabilidad.

\section{INTRODUÇÃO}

As enteroparasitoses estão amplamente difundidas pelo mundo e constituem-se um grande agravo de saúde pública, em especial, pelo seu amplo alcance de disfunções e distúrbios orgânicos que podem acarretar aos indivíduos (SILVA MBMA, 2018). Dentre as alterações biológicas de grande importância estão a anemia e distúrbios intestinais, esses fatores podem culminar em uma desnutrição consequente da má absorção disponibilizada pela presença de enteroparasitas que atuam como fatores co-adjuvantes para manutenção ou agravamento deste tipo de quadro clínico (VIANA ML, et al., 2017).

Na Região Norte do Brasil a ocorrência de enteroparasitoses é elevada, particularmente nas áreas com deficiência de saneamento básico (CASTRO TJ, et al., 2011). Essas áreas se caracterizam como aglomerado populacional, situação pela qual gera-se ambiente peculiar, com efeitos sobre a incidência e prevalência de doenças e na deficitária disponibilidade dos serviços para o atendimento à saúde (MARINHO GL, et al., 2019; RODRIGUES PCO, 2016).

Conforme Cardoso BA, et al. (2018), na Amazônia brasileira são inúmeros os estudos que demonstram uma elevada infestação de parasitoses intestinais, porém, no que tange ao estado do Amapá, ainda são poucos os estudos que determinem a prevalência destes enteroparasitas, principalmente no tocante as suas populações mais vulneráveis como as ribeirinhas.

Adicionalmente, no âmbito Amazônico Brasileiro, a anemia ainda é uma patologia de grande prevalência, sendo potencializado pelas enteroparasitoses, das quais, são responsáveis por reduzir em até $20 \%$ o total de ferro ingerido através da dieta, acarretando uma grave desordem nutricional, em especial, nas crianças menores de 5 anos (CASTRO TJ, et al., 2011).

A anemia por deficiência de ferro ainda é um dos principais problemas em saúde pública do norte do Brasil, situação muito peculiar entre comunidades rurais e vulneráveis as condições socioeconômicas e de saneamento básico (GOMES KM, et al., 2016; VIANA ML, et al., 2017).

A anemia ferropriva se caracteriza pela diminuição da hemoglobina que se encontra na corrente sanguínea, e pode ser observada a partir de sua redução pelo número de células eritrocitárias circulantes no sangue, o que acarreta uma baixa oxigenação tecidual, podendo também, possuir ligação intercorrente relacionada a morbimortalidade, devido a facilidade com que este estado clínico é passível da instalação de processos infecciosos (FARIA FC, et al., 2018).

Outra relação importante da anemia é sua associação com as parasitoses intestinais, o que se constitui em tema de grande interesse para saúde pública, principalmente ao correlacionar com crianças em idade escolar (SILVA RSB, 2019). Nesta faixa etária, a presença dos enteroparasitas é decorrente da baixa condição higiênica-sanitária e de amadurecimento do sistema imunológico, que geralmente determinam a 
causa ou agravamento dos casos de anemia, sendo as manifestações clinicas proporcionais ao nível de infestação parasitaria albergada pelo indivíduo (ALVES FV, et al., 2014; CASTRO TJ, et al., 2011). Dentre as principais consequências e manifestações clínicas podemos citar: diarreia crônica, má absorção, anemia ferropriva, baixa capacidade de concentração e dificuldades no aprendizado (SILVA MBMA, 2018).

As parasitoses intestinais e suas patologias associadas são comuns entre comunidades ribeirinhas e rurais, áreas pouco favorecidas em termos de renda familiar e saneamento básico, estrato das desigualdades socioeconômicas reconhecidamente latentes no país (VIANA ML, et al., 2017; SILVA AMB, et al., 2014). Esses fatores influenciam diretamente para que esta moléstia seja ainda mais pungente entre as comunidades ribeirinhas amazonidas, principalmente entre menores de 5 anos, idosos e mulheres gestantes, período onde a anemia e desnutrição tendem a gerar maiores sequelas (SILVA AMB, et al., 2014).

Diante disso há escassez de dados epidemiológicos em populações ribeirinhas do estado do Amapá, incluindo fatores de risco e morbimortalidade, tornando-se tema de interesse e relevância no auxílio ao mapeamento da situação das enteroparasitoses no estado. Diante do exposto, esta pesquisa objetivou verificar a prevalência de parasitoses intestinais e sua relação com a anemia e estado nutricional de moradores de uma comunidade ribeirinha do Amapá.

\section{MÉTODOS}

Estudo transversal realizado em uma comunidade do estado do Amapá, região amazônica brasileira. Distante trinta minutos de Macapá. A comunidade é conhecida pela beleza turística de balneários particulares e abertos ao público. A localidade foi selecionada pela diferença do nível socioeconômico e ambiental, e, pela presença de pessoas que vivem em condições de vulnerabilidade social. A Comunidade possui influência da maré, observada principalmente durante o período chuvoso, a largura do rio é de aproximadamente 50 metros e sua profundidade varia de 4 a 25 metros.

\section{Sujeito da pesquisa}

A pesquisa foi focada na população de uma comunidade do Amapá, sendo atendidos por demanda espontânea. Adicionalmente, foi atendido todas as faixas etária de idade da população. Para fins de análise, foram consideradas quatro categorias por faixa etária: 0-2 anos, 3-10 anos, 11-18 anos e acima de 18 anos completos. Segundo o líder da comunidade a vila possui em torno de 300 moradores, destes, 128 são eleitores aptos para as eleições de 2020, sendo esse público residentes e domiciliados as margens do rio.

\section{Critérios de seleção}

Os participantes foram obrigados a atender aos seguintes critérios de inclusão: (1) formulário do termo de consentimento livre e esclarecido assinado, (2) assinatura do termo de assentimento livre e esclarecido para pais ou responsáveis pelos menores de 18 anos, (3) ser nativo da área de estudo, (4) preenchimento do formulário de coleta das informações epidemiológicas (5) consentir na coleta de sangue e (6) fornecer amostras fecais.

Como critérios de exclusão, não foram aceitos na pesquisa as pessoas de trânsito na comunidade, que apresentaram alguma patologia que, de acordo com exame médico prévio, não possa participar da pesquisa e que não concordem com a assinatura do termo de consentimento e/ou assentimento livre e esclarecido, além do preenchimento das informações epidemiológicas.

\section{Coleta e período de estudo}

A coleta ocorreu em uma escola pública da comunidade, na ocasião, aplicou-se o formulário epidemiológico. As amostras fecais foram coletadas e devidamente acondicionadas em coletores plásticos descartáveis contendo como fixador solução de formol a $10 \%$. A pesquisa foi focada na população e os atendimentos ocorreram por demanda espontânea na comunidade. O período da coleta de dados ocorreu durante seis meses, com intervalo compreendido entre os meses de janeiro a junho de 2019.

\section{Instrumento de coleta de dados}

Para subsidiar os resultados, foi elaborado um formulário epidemiológico contendo questões fechadas e abertas pertinentes ao objetivo do estudo, através de entrevista interpessoal com perguntas de cunho 
socioeconômico, higiênico-sanitário e de morbidades pregressas. Para os menores de 18 anos, a entrevista foi realizada em conjunto com os pais e/ou responsáveis legais, ao término de cada pergunta, foi deixado um espaço para o registro de possíveis informações não expressas no formulário e verbalizadas pelo entrevistado da pesquisa.

\section{Coleta e exame de sangue}

As amostras de sangue foram coletadas por punção venosa, sendo coletados oito mililitros de sangue venoso de cada paciente. Quatro mililitros foram distribuídos em um tubo contendo EDTA (ácido etilenodiamina tetraacetico) (Beckton \& Dickson, EUA) para identificar os níveis de hemoglobina e realizar análises hematológicas e quatro mililitros foram distribuídos em um tubo sem EDTA para análise da ferritina.

\section{Hemograma}

O hemograma compreende a contagem das células do sangue periférico (hemácias, leucócitos e plaquetas) e a contagem diferencial dos tipos leucocitários, além da quantidade dos valores da hemoglobina e do hematócrito e ainda o cálculo dos índices hematimétricos. Nessa pesquisa foi utilizado o equipamento automatizado (Mindray-BC-3000plus).

O equipamento possibilita a contagem de leucócitos totais (WBC), percentual diferencial de leucócitos em cinco partes, quantificação de eritrócitos (RBC), quantificação de plaquetas (PLT), dosagem de hemoglobina (HGB), hematócrito (HCT), volume corpuscular médio (VCM), hemoglobina corpuscular média (HCM), concentração de hemoglobina corpuscular média ( $\mathrm{CHCM})$, coeficiente de variação da distribuição de eritrócitos (RDWCV), desvio padrão da distribuição de eritrócitos (RDWSD) e volume plaquetário médio (VPM) (NAOUM PC, 2011).

\section{Dosagem de hemoglobina}

A anemia foi avaliada de acordo com os padrões de diagnóstico recomendados pela Organização Mundial da Saúde através de índices de hematócrito e hemoglobina com pequenas variações de acordo com a idade e sexo.

\section{Dosagem de ferritina}

As dosagens de ferritina foram feitas a partir de amostras de soro ou plasma dos pacientes pelo método de turbidimetria. As dosagens bioquímicas de ferritina foram feitas utilizando kit para determinação de ferritina por turbidimetria (Analisa, Gold Analisa Diagnóstica Ltda).

\section{Diagnóstico fecal}

As amostras fecais foram preparadas usando a técnica e/ou métodos a fresco, direto e de Hoffman-PonsJaner. Para cada amostra, duas lâminas foram examinadas para detecção de parasitas, utilizando microscopia óptica (Nikon, Japão) com ampliações de 100X e 400X. As análises foram realizadas no Laboratório de Análises Clinicas (LAC) e no Laboratório de estudos morfofuncionais e parasitário (LEMP) da Universidade Federal do Amapá.

\section{Medidas Antropométricas}

Os participantes foram pesados e medidos descalços e sem qualquer acessório, em posição ortostática, com os pés paralelos, braços estendidos ao longo do corpo e cabeça ereta. Para a medida do peso, utilizouse uma balança eletrônica e mecânica, para mensuração da altura, uma fita métrica com marcações em milímetros, afixada em uma superfície plana, em ângulo de 90ํำ com o chão e através de balança mecânica.

Para determinar o índice de massa corporal (IMC), calculou-se o quociente do peso em quilogramas pelo quadrado da altura em metros: peso $(\mathrm{kg}) /$ estatura $(\mathrm{m})^{2}$, sendo utilizados os pontos de corte para critérios de classificação adotados pela ABESO (2016).

\section{Análise Estatística e considerações éticas}

Os dados obtidos foram armazenados em um programa de banco de dados da Microsoft Office Access 2016, sendo organizados em tabelas de contingência 2x2 relacionando a prevalência global de indivíduos 
parasitados, sexo, faixa etária e dados sócio epidemiológicos. Os valores foram avaliados pela estatística descritiva, sendo utilizado as frequências absolutas e relativas das variáveis de maior relevância para a construção gráficos e tabelas. O período de estudo ocorreu entre os meses de janeiro a junho de 2019, cuja aprovação pelo Comitê de Ética-CEP/UNIFAP, conforme parecer: 3.003.159.

\section{RESULTADOS}

Foram analisados 136 residentes da comunidade, com idades entre 0 a 68 anos, destes, 64 (47,05\%) eram homens e 72 (52,95\%) mulheres, onde, a princípio, foram estudados apenas os índices de hemoglobina, o que demonstrou uma elevada parcela de indivíduos com resultados fora do valor de referência.

Adicionalmente, das 136 amostras, um total de 65 apresentaram índices hematimétricos sugestivos de anemia, ou seja, uma prevalência de $47,8 \%$ de casos sugestivos para anemia, sendo destes, $75,4 \%$ (49/65) de crianças e adolescentes e apenas 24,6\% (16/65) com idade acima de 18 anos (Gráfico 1).

Gráfico 1 - Dados de prevalência em índices hematimétricos sugestivos para anemia em relação a faixa etária.

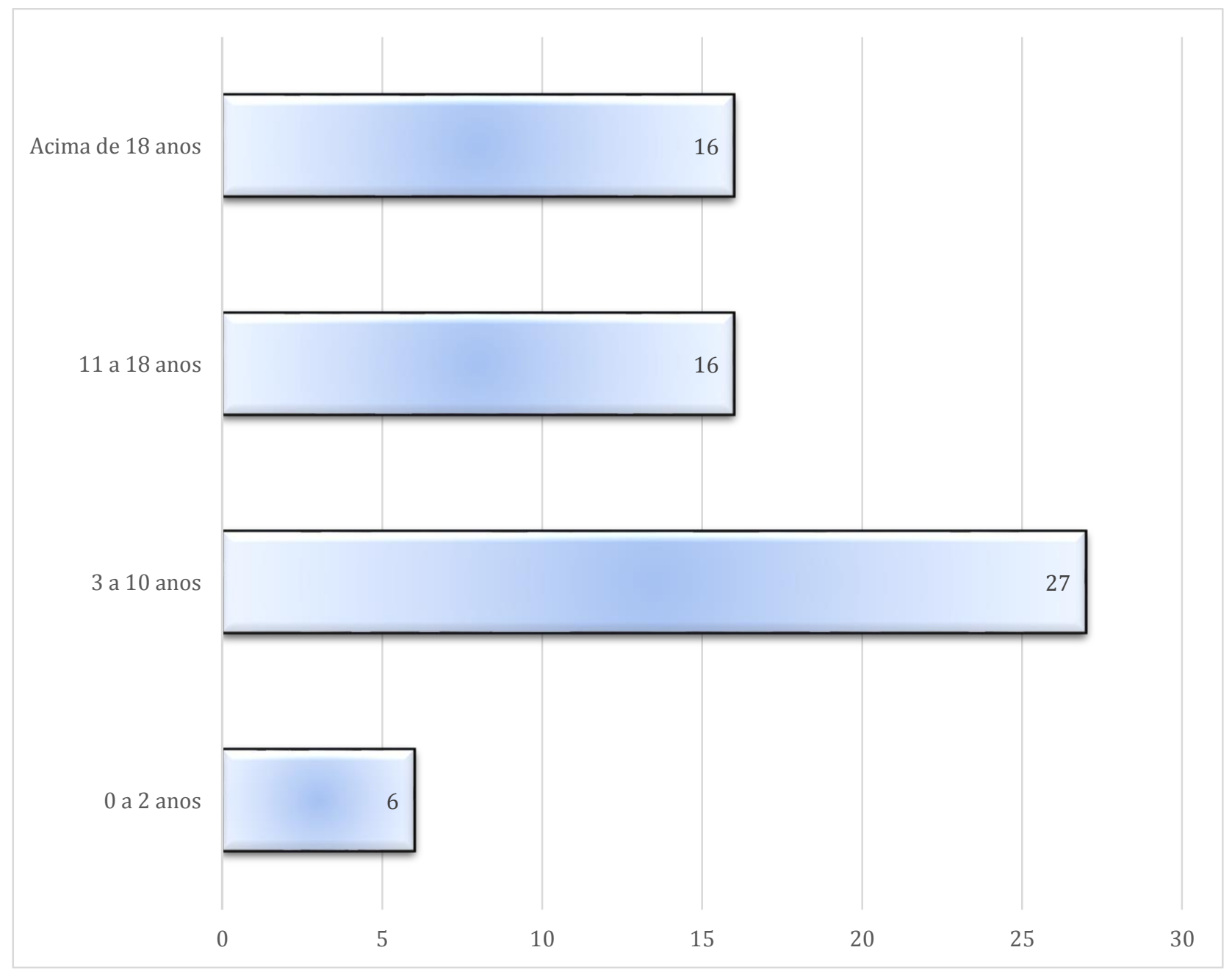

Fonte: Júnior RCM, et. al., 2019.

$\mathrm{Na}$ avaliação dos demais índices hematimétricos observou-se uma relação de 41,53\% (27/65) de valores de volume corpuscular médio (VCM) e 50,76\% (33/65) de hemoglobina corpuscular média (HCM) que estavam abaixo dos valores de referência de 78,0fL e 25,0pg, respectivamente, (valores limítrofes inferiores) (Gráfico 2). 
Gráfico 2 - Prevalência dos valores hematimétricos em relação a hemoglobina

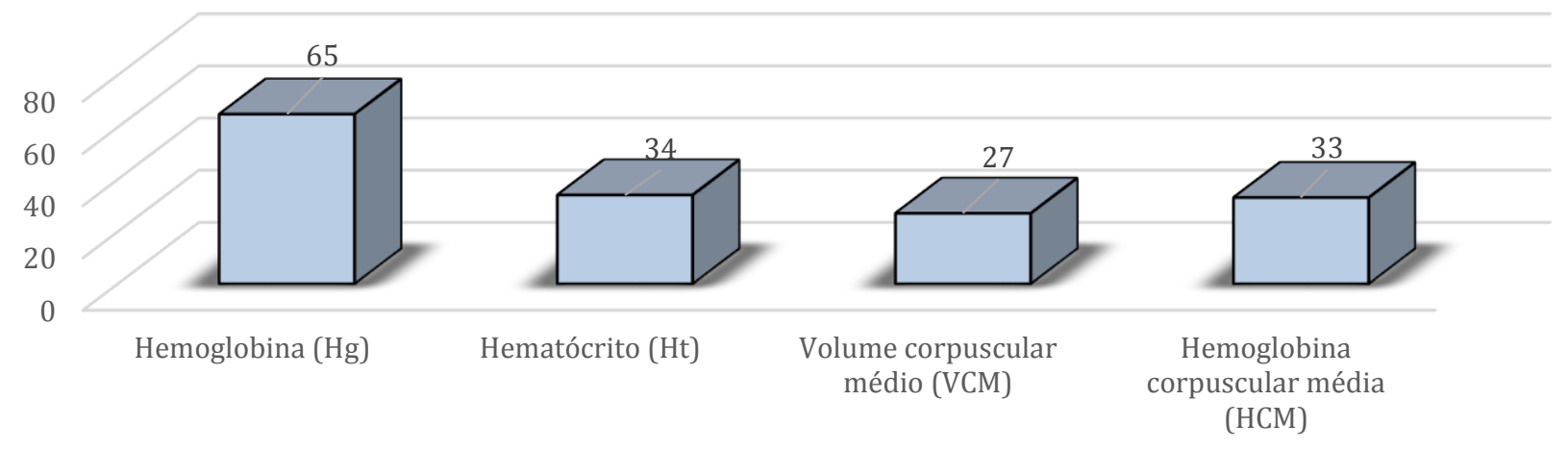

Fonte: Júnior RCM, et. al., 2019.

Nos resultados qualitativos de ferritina sérica por quimiluminescência, houve variantes entre 5,6 a 153 $\mathrm{ng} / \mathrm{mL}$, destes, foram detectados 3 casos de anemia ferropriva, todos de adultos do sexo feminino, sendo uma no período menstrual e duas no período gestacional. Após análise desses dados, foram observados valores de índice de massa corpórea (IMC) de todos os 65 indivíduos com indicativos de hemoglobina deficitária e sugestivos de anemia, sendo 56,9\% (37/65). Adicionalmente, as variáveis epidemiológicas e coprológicas com e sem anemia estão expressos na (Tabela 1).

Tabela 1 - Dados epidemiológicos e resultados coprológicos com e sem anemia.

\begin{tabular}{ccc}
\hline Variáveis & Com anemia n(\%) & Sem anemia n(\%) \\
\hline Peso & $37(100 \%)$ & $0(0 \%)$ \\
Baixo peso & $16(44,4 \%)$ & $20(55,6 \%)$ \\
Peso normal & $12(19 \%)$ & $51(81 \%)$ \\
Acima do peso & & $36(44,4 \%)$ \\
\hline Modalidade de parasitismo & $45(55,6 \%)$ & $29(59,2 \%)$ \\
\hline Poliparasitismo & $20(40,8 \%)$ & $6(100 \%)$ \\
Monoparasitismo & - & \\
Negativo & & $65(53,7 \%)$ \\
\hline Ocorrências de parasitoses & $56(46,3 \%)$ & - \\
\hline Protozoários & $9(100 \%)$ & $0(0 \%)$ \\
Helmintos & - & $0(0 \%)$ \\
Negativo & & \\
\hline Protozoários & $16(100 \%)$ & $0(0 \%)$ \\
\hline Giardia intestinalis & $9(100 \%)$ & $0(0 \%)$ \\
\hline Helmintos & & $0(0 \%)$ \\
\hline Ascaris lumbricoides & $6(100 \%)$ &
\end{tabular}

Fonte: Júnior RCM, et. al., 2019. 
$\mathrm{Na}$ (Tabela 2), foi observado os aspectos epidemiológicos, socioeconômicos e higiênico-sanitários do estudo, sendo $95,6 \%$ (130/136) de ocorrências de indivíduos parasitados, com destaque ao poliparasitismo $59,6 \%(81 / 136)$, prevalência para o sexo feminino 50,7\% (69/136), faixa etária de $3-10$ anos $41,9 \%(57 / 136)$, ensino fundamental incompleto $62,5 \%$ (85/136), que recebe bolsa ou pensão governamental $73,5 \%(100 / 136)$, com abastecimento de água por poço amazonas 60,3\% (82/136). Complementarmente, essa tabela apresenta outras variáveis relevantes ao estudo. 


\section{Revista Eletrônica Acervo Saúde / Electronic Journal Collection Health ISSN 2178-2091}

Tabela 2 - Aspectos epidemiológicos, socioeconômicos e higiênico-sanitários.

\begin{tabular}{|c|c|c|}
\hline Variável & Parasitados (n) & Frequência (\%) \\
\hline \multicolumn{3}{|l|}{ Ocorrências } \\
\hline Positivo & 130 & $95,6 \%$ \\
\hline \multicolumn{3}{|l|}{ Intensidade do Parasitismo } \\
\hline Monoparasitismo & 49 & $36 \%$ \\
\hline Poliparasitismo & 81 & $59,6 \%$ \\
\hline \multicolumn{3}{|l|}{ Sexo } \\
\hline Masculino & 61 & $44,9 \%$ \\
\hline Feminino & 69 & $50,7 \%$ \\
\hline \multicolumn{3}{|l|}{ Faixa Etária } \\
\hline $0-2$ anos & 12 & $8,8 \%$ \\
\hline 3-10 anos & 57 & $41,9 \%$ \\
\hline 11-18 anos & 30 & $22,1 \%$ \\
\hline Acima de 18 anos & 31 & $22,8 \%$ \\
\hline \multicolumn{3}{|l|}{ Escolaridade do responsável } \\
\hline Ensino fundamental incompleto & 85 & $62,5 \%$ \\
\hline Ensino fundamental completo & 25 & $18,3 \%$ \\
\hline Ensino médio incompleto & 11 & $8,1 \%$ \\
\hline Ensino médio completo & 7 & $5,1 \%$ \\
\hline Ensino superior & 2 & $1,5 \%$ \\
\hline \multicolumn{3}{|c|}{ Recebe bolsa ou pensão governamental } \\
\hline Sim & 100 & $73,5 \%$ \\
\hline Não & 30 & $22,1 \%$ \\
\hline \multicolumn{3}{|l|}{ Abastecimento de água } \\
\hline Água de poço artesiano & 7 & $5,1 \%$ \\
\hline Água de poço amazonas & 82 & $60,3 \%$ \\
\hline Água do rio & 41 & $30,1 \%$ \\
\hline \multicolumn{3}{|l|}{ Água de consumo } \\
\hline Filtrada ou fervida & 15 & $11 \%$ \\
\hline Tratada com hipoclorito & 92 & $67,6 \%$ \\
\hline Sem tratamento & 23 & $16,9 \%$ \\
\hline
\end{tabular}

REAS/EJCH | Vol.12(5) | e2841 | DOI: https://doi.org/10.25248/reas.e2841.2020 Página 8 de 13 


\section{Revista Eletrônica Acervo Saúde / Electronic Journal Collection Health ISSN 2178-2091}

\begin{tabular}{|c|c|c|}
\hline \multicolumn{3}{|c|}{ Água usada na higienização de frutas e verduras } \\
\hline Filtrada ou fervida & 4 & $2,9 \%$ \\
\hline Tratada com hipoclorito & 88 & $64,7 \%$ \\
\hline Sem tratamento & 38 & 27,9 \\
\hline \multicolumn{3}{|l|}{ Água usada para preparo de alimentos } \\
\hline Água de poço tratada com hipoclorito & 26 & $19,1 \%$ \\
\hline Água de poço sem tratamento & 60 & $44,1 \%$ \\
\hline Água do rio tratada com hipoclorito & 13 & $9,6 \%$ \\
\hline Água do rio sem tratamento & 27 & $19,9 \%$ \\
\hline Água filtrada ou fervida & 4 & $2,9 \%$ \\
\hline \multicolumn{3}{|l|}{ Destinação do lixo } \\
\hline Recolhido & 115 & $84,6 \%$ \\
\hline Queimado & 10 & $7,4 \%$ \\
\hline Enterrado & 5 & $3,6 \%$ \\
\hline \multicolumn{3}{|l|}{ Hábito de andar descalço } \\
\hline Sim & 44 & $32,4 \%$ \\
\hline Não & 86 & $63,2 \%$ \\
\hline \multicolumn{3}{|c|}{ Qual o destino do esgotamento da privada } \\
\hline Fossa séptica & 64 & $47 \%$ \\
\hline Fossa seca & 43 & $31,6 \%$ \\
\hline O rio & 23 & $16,9 \%$ \\
\hline \multicolumn{3}{|l|}{ Núcleo familiar } \\
\hline$\leq 3$ & 50 & $36,8 \%$ \\
\hline$\geq 4$ & 80 & $58,8 \%$ \\
\hline \multicolumn{3}{|l|}{ Morbidades pregressas } \\
\hline Diarreia & 44 & $32,35 \%$ \\
\hline Sangue nas fezes & - & - \\
\hline Dor abdominal & - & - \\
\hline Eliminação de vermes & - & - \\
\hline Não apresenta & 86 & $63,2 \%$ \\
\hline
\end{tabular}

Fonte: Júnior RCM, et. al., 2019. 


\section{DISCUSSÃO}

Os perfis epidemiológicos das parasitoses intestinais são sempre muito variáveis, geralmente potencializados pela carência da rede de saneamento básico e abastecimento de água potável (ALVES FV, et al., 2014). Conforme os estudos de Santos DN, et al. (2017) a ocorrência de parasitas intestinais e consequente anemia possuem sempre grande relevância para a saúde pública, principalmente quando os agentes envolvidos são de comunidades isoladas, onde o acesso a saúde e saneamento básico são quase inexistentes.

Adicionalmente, a pesquisa evidenciou 52,30\% (34/65) dos hematócritos como sugestivos para anemia (abaixo da normalidade), e após associação com as demais análises dos dados hematimétricos, observouse que esse perfil retrata um indicativo de uma anemia microcítica e hipocrômica, acompanhada de moderada anisocitose (FARIA FC, et al., 2018).

Os dados, além de observáveis nos esfregaços sanguíneos, também puderam ser confirmados através da morfologia dos eritrócitos, sendo a contagem de reticulócitos de grande auxilio na análise do tipo de anemia que aflige os indivíduos, pois, através dos reticulócitos é possível descartar uma incapacidade na produção medular, uma hemólise ou perda sanguínea recente (NAOUM PC, 2011; GOMES KM, et al., 2016).

Apesar dos resultados não demonstrarem uma anemia ferropriva os dados demonstram que $47,8 \%$ (65/136) da população apresenta uma anemia microcítica e hipocrômica diferenciados pelas baixas taxas nos índices hematimétricos, essa redução de hemoglobina nos eritrócitos ou do conteúdo de hemoglobina abaixo do nível fisiológico necessário, gera um quadro que causam alterações visíveis na morfologia dos eritrócitos, dando assim, munição para o diagnóstico e a descoberta do tipo de anemia prevalente (SANTOS SLF, et al., 2016).

As anemias microcíticas e hipocrômicas são resultantes de várias condições patológicas e são as mais prevalentes na população brasileira (GOMES KM, et al., 2016; SANTOS SLF, et al., 2016). Estudos dessa natureza são importantes e de extrema relevância, pois criam indicadores da população quanto as ocorrências dessas anemias, auxiliando no diagnóstico e sugerindo critérios de acompanhamento clínico mais especifico, eficiente e de qualidade (SANTOS SLF, et al., 2016; GOMES KM, et. al., 2016).

Nesse contexto, associado ao conhecimento de que nos países em desenvolvimento é nítido o agravo da desnutrição, e este reflexo é facilmente observável no Brasil, onde, essa carência nutricional acaba se tornando predominante em regiões como a do norte do país, que possui papel fundamental na cadencia da desnutrição infantil (CASTRO TJ, et al., 2011). Essa grave situação de precariedade nutricional é ainda mais agravante nas áreas rurais em especial, nas comunidades ribeirinhas distantes do acesso e dos serviços de saúde, onde a vulnerabilidade social prevalece constantemente (GOMES KM, et al., 2016; VISSER S, et al., 2011).

Nesse estudo o acesso a água tratada e as condições de higiene da população foram deficitários. Os dados antropométricos coletados na população em questão descrevem um grande número de indivíduos com índice de massa corpórea abaixo do peso ideal, demonstrando uma elevada desnutrição, principalmente nos menores em idade escolar. Essa falta de nutrientes alimentares adequados pode estar associada a diminuição da síntese de hemoglobina ou provável deficiência de ferro sérico, tornando-se assim, uma variável importante na descoberta da fonte da anemia (FARIA FC, et al., 2018).

Durante análise parasitária, observou-se que crianças em idade escolar foram mais susceptíveis as parasitoses gastrointestinais o que provavelmente se deu devido à falta de higiene e condições ambientais favoráveis (SOUZA FR, et al., 2018). É importante ressaltar que infecções por parasitas intestinais possuem um tratamento rápido, eficiente e de baixo custo, e nestas comunidades, como a comunidade ribeirinha em questão, é comum durante ações de cunho social, haver a distribuição de medicamentos antiparasitários, além de distribuição de hipoclorito, para o tratamento da água de consumo, fatores importantes que podem diminuir o número de infecções e o número de pessoas contaminadas (VIANA ML, et al., 2017).

Nesse sentido, é importante frisar que, apesar de haver tratamento, o efeito não é duradouro e as infecções acabam sendo frequentes por conta das condições socioambientais permanecerem as mesmas, fazendo com 
que as prevalências das infecções entre os parasitas intestinais se mantenham sempre equivalentes (RODRIGUES PCO, et. al., 2016). Adicionalmente as regiões ribeirinhas são de difícil acesso e acabam por possuir dificuldades de diagnóstico e consequente retardo no tratamento, o que leva a um ciclo vicioso da cadeia de infecção. Os valores de infecções parasitárias observados neste estudo apenas corroboram e confirmam os altos índices de prevalência de enteroparasitas na Amazônia como um todo (SILVA AMB, et al., 2014).

A observância dos fatores de risco na população em relação as infecções parasitárias se dão através da análise dos agentes etiológicos, demonstrando que a contaminação oro-fecal é o mecanismo de transmissão comum, tanto para os enterocomensais como a Endolimax nana e Entamoeba coli, quanto para os enteroprotozoários patogênicos como Giardia intestinalis e Entamoeba histolytica/E. díspar. Portanto, essa relação de similaridade entre o mecanismo de contaminação e infecções enteroparasitárias serve como sinalizador das condições sanitárias e sociais da comunidade (CALDERARO A, et al., 2014).

Entre os protozoários patogênicos mais frequentes, a pesquisa evidencia a presença de E. histolytica/E. díspar. Este agente causa a amebíase, considerado a segunda principal causa de morte entre as doenças parasitárias, entre as suas manifestações clínicas mais frequentes estão a diarreia e a colite amebiana aguda, assumindo formas invasivas intestinais e extraintestinais (CARDOSO BA, et al., 2018).

Esses sinais clínicos corroboram para os achados de diarreia nos indivíduos entrevistados. Todavia, a infecção E. histolytica/E. Dispar é hipotética, levando em consideração que este parasita patogênico ( $E$. histolytica) necessita de laboratório de diagnóstico molecular para a diferenciação real entre estas espécies (patogênica e não patogênica) (RODRIGUES PCO, et al., 2016).

Adicionalmente, a Giárdia intestinalis foi o protozoário patogênico que ganhou a maior relevância no estudo. Apesar de sua presença corresponder apenas 6,6\% dos indivíduos analisados. Para Silva RSB, et al. (2019) a presença deste parasita sinaliza que a água está abaixo das necessidades ideais de pureza.

Em complemento, Santos DN, et al. (2017), informa que a contaminação por $G$. intestinalis possui forte ligação com água e areia contaminada por dejetos de animais portadores deste parasita (em geral, cães e gatos). Vale ressaltar que, entre os protozoários, este é o que permanece viável por mais tempo no ambiente externo e resiste à cloração usual de água.

Entre as espécies de helmintos encontradas, verificou-se a presença de Ascaris lumbricoides 4,4\%, Trichuris trichiura 1,5\% e Ancilostomídeos 0,7\% geohelmintos parasitas, que podem causar infecção no intestino delgado de humanos com sintomas de anemia grave (ARAÚJO MM, et al., 2019). A presença destes parasitas demonstra que a localidade ribeirinha apresenta condições ambientais favoráveis, propicia ao desenvolvimento destes parasitas e contribui para sua manutenção e disseminação.

Conforme Mori FMLR, et al. (2016), é comum observarmos a alta prevalência destes enteroparasitas nas regiões menos desenvolvidas, devido às condições higiênico-sanitárias e socioeconômicas serem insatisfatórias. Estas condições descritas formam uma situação ecoepidemiológica que favorecem a transmissão e reinfecção destes parasitas, o que justifica a prevalência constante destas parasitoses nesse estudo e diversos outros na Amazônia com público semelhante (FERNANDES NS, et al., 2014).

Os resultados encontrados neste estudo demonstram uma elevada prevalência de anemia em crianças e jovens, com necessidade de acompanhamento médico e nutricional, além de exames laboratoriais complementares para determinar se está anemia se deve à deficiência de ferro (PICANÇO NJA, et al., 2019). Quanto ao quadro de alta infecção enteroparasitária, podemos observar que estão diretamente proporcionais as condições higiênico-sanitárias deficitárias.

A pesquisa evidenciou predominância das protozoonoses frente às helmintíases e da abrangência dos fatores associados (BACELAR PAA, et al., 2018). Adicionalmente, o estudo apresenta limitações inerentes a estudos transversais, não sendo possível estabelecer relação de causalidade, baseado na complexidade das variáveis de risco relacionadas à grande diversidade destes em diferentes perfis populacionais (COJULUN AC, et al., 2015; BACELAR PAA, et al., 2018; PICANÇO NJA, et al., 2019). Entretanto, essas limitações não comprometem os resultados obtidos, sendo suficientes para atingir os objetivos do estudo. 
É importante destacar que o quantitativo de casos positivos investigados, demonstram uma necessidade de implantação de programas de orientação e conscientização quanto ao tratamento, saneamento e educação, tanto domiciliares como peridomiciliares, que venham a estimular hábitos higiênicos e sanitários adequados que garantam melhorias na qualidade de vida de todos os moradores da comunidade, especialmente quanto a redução de infecção por parasitas intestinais.

\section{CONCLUSÃO}

Com base nos resultados, é evidente a elevada prevalência de parasitoses intestinais na comunidade estudada, além de alta prevalência de crianças anêmicas e abaixo do peso, fato observável através de hábitos diários e cuidados inadequados para com a água e higienização de alimentos. A prevalência de enteroparasitoses associada a anemia foi mais frequente entre mulheres e crianças na faixa etária de 3 a 10 anos, sendo necessárias iniciativas governamentais de educação em saúde com propostas de implantar melhorias nas condições básicas de educação e saneamento, além do incentivo a conscientização populacional sobre os riscos de transmissão das enteroparasitoses, tendo como intuito minimizar as taxas de infecção desses agentes, gerando melhor condição na qualidade de vida da comunidade em questão.

\section{AGRADECIMENTOS}

Nossos agradecimentos a gentil aquiescência do líder comunitário que facilitou a nossa inserção na comunidade, dos moradores que consentiram a realização da pesquisa, a Universidade Federal do Amapá (UNIFAP), através de seus laboratórios e equipamentos, nos possibilitando a concretização deste projeto. Adicionalmente, agradecemos a Pró-reitoria de extensão e ações comunitárias da Universidade Federal do Amapá, que por meio de seu departamento de extensão, fomentou esta pesquisa após seleção através de seu edital 001/2019 - DEX/PROEAC para escolha de projetos e concessão de bolsa e auxílio financeiro ao pesquisador, sendo o projeto registrado sob o código PJ032-2019.

\section{REFERÊNCIAS}

1. ABESO. Associação Brasileira para o Estudo da Obesidade e da Síndrome Metabólica - 4.ed. - São Paulo, SP. 2016.

2. ALVES FV, et al. Aspectos epidemiológicos das enteroparasitoses em crianças domiciliadas em um assentamento rural no nordeste brasileiro. Revista Eletrônica Acervo Saúde, 2014; 6(3): 666-676.

3. ARAÚJO MM, et al. Prevalência de enteroparasitoses na região Norte de Mato Grosso. Nativa- revista de ciências sociais do norte de Mato Grosso, 2019; 8(2): 90-95.

4. BACELAR PAA, et al. Parasitoses intestinais e fatores associados no estado do Piauí: uma revisão integrativa. Revista Eletrônica Acervo Saúde, 2018; 10(4): 1802-1809.

5. CALDERARO A, et al. Intestinal parasitoses in a tertiary-care hospital located in a non-endemic setting during 20062010. BMC Infectious Diseases, 2014; 14(264): 2-9.

6. CARDOSO BA, et al. Populações negligenciadas e fatores socioeducacionais: aplicação de um guia metodológico para tuberculose e parasitoses intestinais. Revista ciência e saberes, 2018 4(Esp): 970-977.

7. CASTRO TJ, et al., Anemia e deficiência de ferro em pré-escolares da Amazônia Ocidental brasileira: prevalência e fatores associados. Caderno de Saúde Pública. 2011;27(1): 131-142.

8. COJULUN AC, et al. Anemia among Children Exposed to Polyparasitism in Coastal Kenya. The American Journal of Tropical Medicine and Hygiene, 2015; 93 (5): 1099-1105.

9. FARIA FC, et. al. A relação de dados antropométricos e hematológicos com a anemia ferropriva em idade jovem. IV Seminário cientifico da FACIG/ II Jornada de Iniciação Cientifica da FACIG; Manhuaçu: Minas Gerais: 2018.

10. FERNANDES NS, et al. Ocorrência de enteroparasitoses em manipuladores de alimentos de restaurantes em Parnaíba, Piauí-Brasil. Rev Patol Trop., 2014; 43(4): 459-469.

11. GOMES KM, et al. Anemia e parasitoses em comunidade ribeirinha da Amazônia Brasileira. Revista Brasileira de Análises Clínicas. 2016; 48(4):389-93.

12. MARINHO GL, et al. Mortalidade infantil de indígenas e não indígenas nas microrregiões do Brasil. Rev. Bras. Enferm. 2019; 72(1): 57-63.

13. MORI, FMRL, et al. Fatores associados a enteroparasitoses em escolares da rede municipal de ensino de Cambé. Revista Semina: Ciências Biológicas e da Saúde, 2016; 37(1): 15-24.

14. NAOUM, PC. Diagnóstico diferencial das anemias microcíticas e hipocrômicas. Revista Brasileira de Análises Clínicas, 2011; 43(2): 160-162. 
15. PICANÇO NJA, et al. Prevalência de enteroparasitoses em usuários na faixa etária de 2 a 12 anos atendidos por uma Unidade de Saúde da Família do município de Belém, Pará. Revista Eletrônica Acervo Saúde. 2019. Vol.Sup.33, e1321.p 1-7.

16. RODRIGUES PCO, et. al. Características socioeconômicas, demográficas e de saúde de escolares residentes em duas comunidades da amazônia meridional brasileira. Revista Ciência e Estudos Acadêmicos de Medicina. 2016; 1(6): 10-23.

17. SANTOS DN, et al., Saberes sobre parasitoses em uma comunidade riberinha. Caçador, 2017; 6(2):.44-56.

18. SANTOS SLF, et al. Utilização dos índices hematimétricos no diagnóstico diferencial de anemias microcíticas. Mostra Científica da Farmácia, 10. Quixadá: Centro Universitário Católico de Quixadá, 2016.

19. SILVA AMB, et al., Ocorrência de enteroparasitoses em comunidades ribeirinhas do Município de Igarapé Miri, Estado do Pará, Brasil. Revista Pan-Amazônica de Saúde, 2014; 5(4): 45-51.

20. SILVA MBM. A Ocorrência de Parasitoses Intestinais em alunos do ensino fundamental na zona rural rede Municipal de São Luís-Maranhão e sua relação com os hábitos alimentares. Dissertação (Mestrado em educação para saúde): Escola Superior de educação de Coimbra (ESTeSC) e a escola Superior de Tecnologia da Saúde (ESEC), Coimbra, 2018.

21. SILVA RSB, et al., Estudo de parasitoses intestinais em moradores de corumbá, Mato Grosso do Sul, Brasil. Revista Ibero-Americana de Ciências Ambientais. 2019; 10(2):109-128.

22. SOUZA FR, et al. Diagnóstico epidemiológico de parasitos intestinais em uma comunidade rural de Ipatinga-MG. Rev. UNINGÁ, 2018; 55(2): 200-213.

23. VIANA ML, et al. Parasitoses intestinais e a inter-relação com os aspectos socioeconômicos de indivíduos residentes em um povoado rural (Rosápolis de Parnaíba-PI). Scientia Plena, 2017;13(8): 1-10.

24. VISSER S, et al. Estudo da associação entre fatores socioambientais e prevalência de parasitose intestinal em área periférica da cidade de Manaus (AM, Brasil). Ciência Saúde Coletiva, 2011; 16(8): 3481-92. 